\title{
BMJ Open Optimising expert dyad performance in acute care settings: a scoping review protocol
}

\author{
Katie Walker (D) , ${ }^{1}$ Maryam Asoodar, ${ }^{1}$ Jenny Rudolph, ${ }^{2}$ Michael Meguerdichian, ${ }^{3}$ \\ Tricia Yusaf, ${ }^{3}$ Kimberly Campbell-Taylor, ${ }^{3}$ Jeroen van Merriënboer ${ }^{1}$
}

To cite: Walker K, Asoodar M, Rudolph J, et al. Optimising expert dyad performance in acute care settings: a scoping review protocol. BMJ Open 2021;11:e047260. doi:10.1136/ bmjopen-2020-047260

- Prepublication history for this paper is available online To view these files, please visit the journal online (http://dx.doi. org/10.1136/bmjopen-2020 047260).

Received 24 November 2020 Accepted 24 June 2021

Check for updates

(C) Author(s) (or their employer(s)) 2021. Re-use permitted under CC BY-NC. No commercial re-use. See rights and permissions. Published by BMJ.

${ }^{1}$ School of Health Professions Education, Maastricht University, Maastricht, The Netherlands ${ }^{2}$ Center for Medical Simulation and Massachusetts General Hospital, Boston, MA, USA

${ }^{3}$ Simulation Center, NYC Health + Hospitals, Bronx, New York, USA

Correspondence to

Katie Walker;

Katie.walker@nychhc.org

\section{ABSTRACT}

Introduction When there is miscommunication and poor coordination between experienced clinician dyads, teamwork suffers. Research on expert learning practices for the smallest team, and arguably the most important team, the healthcare dyad, is limited. The objective of this study is to map the extent and range of evidence available on learning practices which experienced dyads use, to achieve excellent performance, and to identify the gaps in effective practice. This will guide future research, policy and practice.

Methods and analysis We are using the JBI methodology for scoping reviews and the Preferred Reporting Items for Systematic Reviews and Meta-analyses extension for Scoping Reviews Extension Fillable Checklist, searching for literature that meets the inclusion criteria. The searches will be conducted using Maastricht University's Libsearch, which includes MEDLINE, Education Resources Information Center and PsycINFO and a second search on Web of Science online databases. We will search grey literature and references of selected sources. Search limits include sources from 2016 to 2021, using English language only. A data extraction tool was developed, and charting will use a thematic analysis approach.

Implications and dissemination This review will be the first to examine the learning practices that experienced dyads use, which ensures excellent performance in acute care settings. The findings will be used to develop best-practices and shared with New York City hospital system. Dissemination will occur through peer-reviewed publications and at healthcare conferences.

\section{INTRODUCTION}

Medical error in healthcare, particularly in the perioperative environment, remains a major cause of morbidity and mortality. WHO in $2019^{1}$ reported that unsafe surgical care procedures cause complications in up to $25 \%$ of patients, resulting in 1 million deaths during or immediately after surgery annually. Anecdotal evidence suggests that when some experienced healthcare dyads are faced with difficult and complex situations, excellent performance is difficult to achieve while other experienced dyads perform with excellence every time. Cooper ${ }^{2}$ specifically suggests the

\section{Strengths and limitations of this study}

This scoping review is the first to examine learning practices of expert healthcare dyads and is unique.

- This review is comprehensive, including all study designs and grey literature from the past 6 years.

- The use of a detailed data extraction form and a transparent, iterative team approach will add rigour to the review.

- The paucity and quality of literature addressing the learning practices of expert healthcare dyads may limit findings.

collaboration between each surgeon-anaesthesiologist dyad in the operating room, is perhaps the most critical element of overall operating room team performance. He says a well-functioning dyad is conducive to safe, effective care. Dysfunctional collaboration can promote unsafe conditions and contribute to an adverse outcome. This may also be true of dominant dyads in other acute care settings such as the emergency room, labour and delivery, critical care and paediatrics. The goal of this scoping review is to understand the learning practices, including communication and coordination rehearsals, that expert dyads use in acute care settings. Learning practices can be defined as the transformative processes of taking in information, internalising it and combining it with previous experience. This changes what we know and builds on what we do. It is based on input, process and reflection. ${ }^{3}$ SebokSeyer et $a l^{t}$ published a scoping review on the approaches for measuring 'interdependent' collaborative performances and found a strong level of interdependence between dyads of trainees and their supervisors. The learning practices of novices and experts and the interdependence between them is very different. $^{5}$ This review is limited to experienced healthcare professionals only. 
Within the largest public hospital system within the USA, a quality assurance meeting is held weekly where selected cases are reviewed. The cases include those where healthcare teams performed as expert teams, and others where team improvement is needed. From the cases discussed at these meetings, it is clear that when there is a breakdown in communication and coordination in healthcare, the failure is usually between two specific members of the healthcare team, the dominant dyad. There is an urgency to understand more about how this breakdown in coordination and communication can be prevented, as it often leads to a fracturing in clinical teams. There is significant research regarding whole team function, but a dearth of literature investigating the learning practices that inform excellence in dyad function. A deeper understanding of human cognitive architecture, ${ }^{6}$ individually and shared ${ }^{7}$ may give insight to optimise dyad communication and coordination.

A scoping review will be conducted, to systematically map current research on learning practices that expert dyads use, in both peer-reviewed journals and grey literature. The review will also identify any existing gaps in knowledge. ${ }^{8}$ This scoping review will target key concepts that underpin learning practices for peak performance in healthcare dyads and clarify working definitions and/ or the conceptual boundaries of learning practices. ${ }^{9}$ In summary, this scoping review aims to explore the breadth or extent of the literature, summarise the evidence and inform future research, ${ }^{10}$ with the overarching objective of providing a 'map' of the available evidence. The authors consider it important to provide this evidence map as it will guide best practice in learning practices that expert dyads use. Finding the answer as to why there is not uniformity in exemplary performance may assist in averting medical errors and assist expert dyadic teams to function more routinely, with excellence. This will achieve the objective of the review and provide recommendations to inform best practice in expert healthcare dyad learning practices.

Unlike a systematic review, scoping reviews do not tend to produce and report results that have been synthesised from multiple evidence sources following a formal process of methodological appraisal to determine the quality of the evidence. Rather, scoping reviews aim to provide an overview or map of the evidence. As a result, an assessment of methodological limitations or risk of bias of the evidence will not be performed. To put it simply, systematic reviews normally inform the development of trustworthy clinical guidelines and recommendations. Scoping reviews are not conducted for this reason but rather to provide an overview of the evidence or to answer questions regarding the nature and diversity of the topic.

In relation to this scoping review, to simplify the many complex communication and coordination errors that plague healthcare dyads in complex situations as a generic communication or coordination error is an overt oversimplification of a complex system phenomenon. ${ }^{11}$ The relational coordination ${ }^{12}$ and communication practices ${ }^{13}$ that optimise the performance of this small pivotal team may better guide the learning practices that lead to expert practice.

Three theoretical perspectives have been selected, that are relevant to this problem: cognitive workload theories, shared mental models or distributed cognition and relational coordination theory.

A deeper understanding of the three theories listed above, and how they interact and complement each other, may assist us to solve the problem of poor performance more effectively. This distinction will guide us to understand learning practices that accelerate expert performance, specifically dyads in acute care settings. As this problem is specific to acute healthcare settings, only expert, interprofessional, dyads will be examined. Student training, single discipline training and ambulatory care teams will not be examined. There is limited research on the learning practices of expert healthcare dyads and so the scoping review will extend to teams rather than only the smallest team, the dyad.

A preliminary search of MEDLINE, the Cochrane Database of Systematic Reviews and JBI Evidence Synthesis was conducted and no current or underway systematic reviews or scoping reviews on the topic were identified.

\section{Study objectives}

The overarching objective of this scoping review is to assess the extent of the literature with respect to identifying and characterising learning practices that expert healthcare dyads use in acute care settings. The inclusion criteria are outlined using the Population, Concept, Context (PCC) framework and include the population of expert healthcare professional dyads/teams, the concept is the learning practices that drive expert performance of experienced healthcare dyads with a focus on workload theories, shared mental models, communication and coordination practices and the context is acute care hospital settings.

There are two questions this scoping review aims to answer. These include what are the learning practices that expert healthcare dyads use to optimise performance in acute settings and second how do expert healthcare dyads develop specific future learning practices to retain optimum performance?

\section{METHODS AND ANALYSIS}

\section{Patient and public involvement}

Patients and the public were not involved in this study.

\section{Eligibility criteria}

Described below are the PCC and Study Designs eligibility criteria.

The participants are experienced healthcare professionals who work in acute settings of any age or any sex. The group must be interprofessional including two or more disciplines. Characteristics of participants who will be excluded are students, novices, healthcare 
professionals who work in non-acute care settings and single profession studies. The concept is learning practices that drive expert performance of experienced healthcare dyads with a focus on workload theories, shared mental models, communication and coordination practices. This also includes learning practices that promote and inform future expertise. The concept exclusion criteria are learning practices for novices and students as well as individual psychomotor skill acquisition. The context includes all acute care settings in hospitals including the operating room, emergency room and critical care environments. Settings in all countries are included and there are no racial or gender-based exclusions. The exclusion criteria are all non-acute care settings including ambulatory care, behavioural health and home care.

\section{Study designs}

This scoping review will consider both experimental and quasi-experimental study designs including randomised controlled trials, non-randomised controlled trials, before-and-after studies and interrupted time-series studies. In addition, analytical observational studies including prospective and retrospective cohort studies, case-control studies and analytical cross-sectional studies will be considered for inclusion. This review will also consider descriptive observational study designs including case series, individual case reports and descriptive crosssectional studies. Qualitative studies will be considered that focus on qualitative data including, but not limited to, designs such as phenomenology, grounded theory, ethnography, qualitative description, action research and feminist research. In addition, systematic reviews that meet the inclusion criteria will be considered, as well as text and opinion papers. The proposed scoping review will be conducted in accordance with the Joanna Briggs Institute (JBI) methodology for scoping reviews. ${ }^{14}$

\section{Search strategy}

The search strategy will aim to locate both published and unpublished studies. The databases to be searched include Maastricht University Libsearch including PsycINFO, MEDLINE, Education Resources Information Center (ERIC) and Web of Science. Sources of unpublished studies/grey literature will be sourced using Canadian Agency for Drugs and Technology in Health (CADTH) Grey Matters tool for health-related grey literature. ${ }^{15}$

The text words contained in the titles and abstracts of relevant articles, and the index terms used to describe the articles were used to develop a full search strategy for the ERIC database (see table 1).

The planned limits included years 2016-2021, worldwide libraries and full-text publications. The search strategy, including all identified keywords and index terms, will be adapted for each included database and/ or information source. The reference list of all included sources of evidence will be screened for additional studies. Studies published in English will be included. Studies published since 2016 will be included as the authors considered a 6-year focus would generate the current evidence on the topic. English language is a limitation as the primary investigator is unilingual.

\section{Source of evidence selection}

Following the search, all identified citations will be collated and uploaded into EndNote X9/June 2019 (Clarivate Analytics, Pennsylvania, USA)) and duplicates removed. Following a pilot test, titles and abstracts will then be screened by two or more independent reviewers

\begin{tabular}{|c|c|c|}
\hline \# & Searches & Results \\
\hline S1 & (“Operating room teams") AND ("performance") & 0 \\
\hline S2 & (“Operating room teams") AND (“practice”) & 0 \\
\hline S3 & (“distributed cognition") AND (“team performance”) & 0 \\
\hline S4 & (“Operating room teams") AND (“learning”) & 0 \\
\hline S5 & (“Cognitive Load”) AND (“dyad performance”) & 0 \\
\hline S6 & "Health dyads" AND “Learning” or "Behavior" & 0 \\
\hline S7 & $\begin{array}{l}\text { ("dyad" OR "surg" dyads" OR "health dyads" OR "surgery") AND (“communicat"” OR "perform"” OR } \\
\text { "coordinat"” OR "expect”" OR "practice*” OR "cognit"” OR "lead") }\end{array}$ & 109 \\
\hline S8 & $\begin{array}{l}\text { ("surg" dyads") AND (“communicat"” OR "perform"” OR "coordinat"” OR "expect”" OR "practice*” OR } \\
\text { "cognit"” OR "lead"”) }\end{array}$ & 4 \\
\hline S9 & 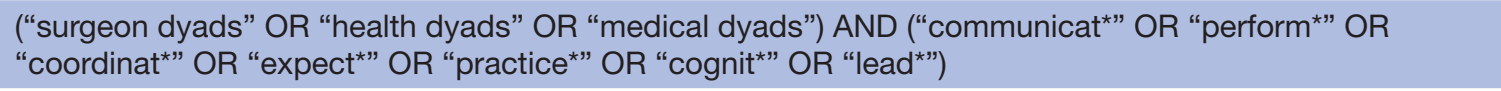 & 6 \\
\hline $\mathrm{S} 10$ & 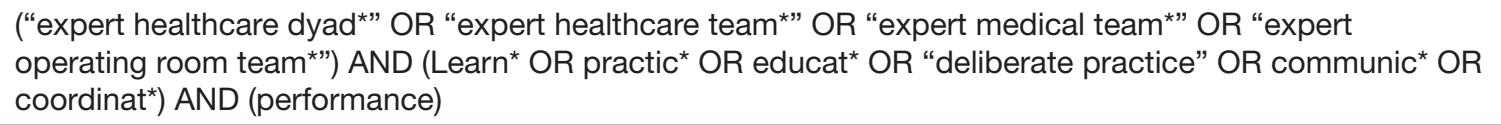 & 1 \\
\hline S11 & $\begin{array}{l}\text { ("healthcare dyad"” OR "healthcare team*" OR "medical team*” OR "operating room team*”) AND } \\
\text { ("Learn*” OR "practic*” OR "educat"” OR "communic*” OR "coordinat"” OR "perform*”) }\end{array}$ & 11 \\
\hline
\end{tabular}


Hidden Dynamics in Healthcare; The Learning Practices that Optimize Expert Dyad Performance in

Acute Care Settings: A Scoping Review 2016-2020

Scoping Review Details

- Name of Reviewer

- Date of Review

EVIDENCE SOURCE DETAILS \& CHARACTERISTICS

1. Citation details: author/s, title, journal, volume, issue, pages

2. Year of publication

3. Location. (please fill in by looking at affiliation of first author)

4. What was the setting of the study?

5. Number of participants enrolled in study

6. What was the profession of participants enrolled in the study? Select at least two.

DETAILS/RESULTS EXTRACTED FROM SOURCE OF EVIDENCE.

1. Which learning practices were identified?

2. How were learning practices conducted?

3. How was learning measured?

4. Was there a specific guide for future learning?

5. What was the research design?

6. Was there agreement or controversy from the authors about the efficacy of the learning practices?

7.Were specific learning practices suggested to optimize future learning?

8. Were there gaps identified to the uptake of effective learning practices?

Figure 1 Data extraction tool.

for assessment against the inclusion criteria for the review. Relevant sources will be retrieved in full with their citation details. The full text of selected citations will then be assessed in detail against the inclusion criteria by two or more independent reviewers. Reasons for exclusion of sources of evidence at full text that do not meet the inclusion criteria will be recorded and reported in the scoping review. Any disagreements that arise between the reviewers at each stage of the selection process will be resolved through discussion with an additional reviewer. The results of the search and the study inclusion process will be reported in full in the final scoping review and presented in a Preferred Reporting Items for Systematic Reviews and Meta-analyses extension for Scoping Reviews flow diagram. ${ }^{16}$

\section{Data extraction}

Data will be extracted from papers included in the scoping review by two or more independent reviewers using a data extraction tool developed by the reviewers. The extraction instrument follows the JBI data extraction tool template with customisation to answer the review objectives. The data extracted will include specific details about the participants, concept, context, study methods and key findings relevant to the review question/s. A draft extraction form is provided (see figure 1). The draft data extraction tool will be modified and revised as necessary during the process of extracting data from each included evidence source. Modifications will be detailed in the scoping review. Any disagreements that arise between the reviewers will be resolved through discussion with an additional reviewer. We will present the contentious manuscript to a third reviewer, and they will present their view on inclusion or exclusion. After thoughtful debate, and a review of the inclusion/exclusion criteria, consensus will be attained. If appropriate, authors of papers will be contacted to request missing or additional data, where required.

\section{Data analysis and presentation}

The evidence presented will directly respond to the review objective and questions. The data will be collected through the survey monkey platform and presented graphically, in diagrammatic form and/or a tabular form. The authors will determine how to best present the data or map and also provide a detailed description. A narrative summary will accompany the tabulated and/or charted results and will describe how the results relate to the reviews objective and questions.

\section{Synthesis of results}

Quantitative (frequencies) and qualitative analyses (generation of descriptions) will be conducted. Thematic analysis will be used to evaluate and categorise study findings, that is, the types of learning practices that optimise expert dyad performance and tools used to measure excellence. We plan that the theoretical underpinnings of cognitive workload, shared mental models and relational coordination will be used as sensitising frameworks and the data will be coded from the findings. Meta-analysis will not be performed. Data will be charted. 


\section{Implications}

The results from this scoping review will grow understanding of the learning practices necessary for excellence in healthcare dyad performance. This in turn may lead to less errors in acute care settings and improved teamwork. This project may offer a cost-effective opportunity to save time and resources by improving the performance of the dyad, therefore advancing the science in this area. To the best of our knowledge, no previous empirical studies have taken this approach.

\section{Dissemination}

This work was conceptualised from the New York City (NYC) Health+Hospitals quality assurance and performance improvement meeting (QAPI) which occurs every week. QAPI is comprised of system board members, senior physicians, nurses and administrative leaders across the system to improve system-based practice in NYC. The review findings will be shared with NYC Health + Hospitals municipal hospital system and the greater New York area. Results, however, may be of interest to hospitals globally who are seeking to improve healthcare errors in acute care settings. Dissemination will occur through peerreviewed publications and healthcare conferences.

\section{Twitter Katie Walker @ubiquitouslinks}

Contributors KW and MA conceptualised the research and drafted the protocol. JvM and JR helped to develop the research idea, conceptualised the study and edited the manuscript. MM, KC-T and TY gave initial ideas, reviewed and edited the manuscript. All authors have made substantive intellectual contributions to the development of this protocol. All have read and approved the manuscript.

Funding The authors have not declared a specific grant for this research from any funding agency in the public, commercial or not-for-profit sectors.

\section{Competing interests None declared.}

Patient and public involvement Patients and/or the public were not involved in the design, or conduct, or reporting, or dissemination plans of this research.

Patient consent for publication Not required.

Provenance and peer review Not commissioned; externally peer reviewed.

Open access This is an open access article distributed in accordance with the Creative Commons Attribution Non Commercial (CC BY-NC 4.0) license, which permits others to distribute, remix, adapt, build upon this work non-commercially, and license their derivative works on different terms, provided the original work is properly cited, appropriate credit is given, any changes made indicated, and the use is non-commercial. See: http://creativecommons.org/licenses/by-nc/4.0/.

ORCID iD

Katie Walker http://orcid.org/0000-0003-2107-8061

\section{REFERENCES}

1 Organization, W.H.O. WHO calls for urgent action to reduce patient harm in healthcare, 2019. Available: https://www.who.int/news/item/ 13-09-2019-who-calls-for-urgent-action-to-reduce-patient-harm-inhealthcare

2 Cooper JB. Critical role of the Surgeon-Anesthesiologist relationship for patient safety. J Am Coll Surg 2018;227:382-6.

3 Bingham T, Conner ML. The new social learning: a guide to transforming organizations through social media. ASTD Press; Berrett-Koehler Publishers, 2010.

4 Sebok-Syer SS, Shaw JM, Asghar F, et al. A scoping review of approaches for measuring 'interdependent' collaborative performances. Med Educ 2021. doi:10.1111/medu.14531. [Epub ahead of print: 07 Apr 2021].

5 Ericsson KA. Acquisition and maintenance of medical expertise: a perspective from the expert-performance approach with deliberate practice. Acad Med 2015;90:1471-86.

6 Sweller J, van Merriënboer JJG, Paas F. Cognitive Architecture and Instructional Design: 20 Years Later. Educ Psychol Rev 2019;31:261-92.

7 Hazlehurst B, McMullen CK, Gorman PN. Distributed cognition in the heart room: how situation awareness arises from coordinated communications during cardiac surgery. J Biomed Inform 2007;40:539-51.

8 Aromataris E MZ, ed. JBI Manual for Evidence Synthesis, 2020.

9 Arksey H, O'Malley L. Scoping studies: towards a methodological framework. Int J Soc Res Methodol 2005;8:19-32.

10 Tricco AC, Soobiah C, Antony J, et al. A scoping review identifies multiple emerging knowledge synthesis methods, but few studies operationalize the method. J Clin Epidemiol 2016;73:19-28.

11 Peters MDJ GC, Mclnerney P, Munn Z. JBI manual for evidence synthesis, 2020.

12 Gittell JH, Godfrey M, Thistlethwaite J. Interprofessional collaborative practice and relational coordination: improving healthcare through relationships. J Interprof Care 2013;27:210-3.

13 Lingard L. Language matters: towards an understanding of silence and humour in medical education. Med Educ 2013;47:40-8.

14 Aromataris E, Munn Z, eds. JBI manual for evidence synthesis. Adelaide, Australia: JBI, 2020.

15 Canadian Agency for Drugs and Technologies in Health (CADTH). Grey matters: a practical tool for searching health-related grey literature. Ottawa, ON Canada: The Agency, 2019.

16 Tricco AC, Lillie E, Zarin W, et al. PRISMA extension for scoping reviews (PRISMA-ScR): checklist and explanation. Ann Intern Med 2018;169:467-73. 